\title{
Bubbles in the Self-Accelerating Universe
}

\author{
Keisuke Izumi $^{a *}$, Kazuya Koyama ${ }^{b \dagger}$, Oriol Pujolàs ${ }^{c \ddagger}$ and Takahiro Tanaka ${ }^{a \S}$ \\ ${ }^{a}$ Department of Physics, Kyoto University, Kyoto 606-8502, Japan \\ ${ }^{b}$ Institute of Cosmology and Gravitation, University of Portsmouth, Portsmouth PO1 2EG, UK and \\ ${ }^{c}$ Center for Cosmology and Particle Physics, New York University New York, NY, 10003, USA
}

\begin{abstract}
We revisit the issue of the stability in the Dvali-Gabadadze-Porrati model, by considering the nucleation of bubbles of the conventional branch within the self-accelerating branch. We construct an instanton describing this process in the thin wall approximation. On one side of the bubble wall, the bulk consists of the exterior of the brane while on the other side it is the interior. The solution requires the presence of a 2-brane (the bubble wall) which induces the transition. However, we show that this instanton cannot be realized as the thin wall limit of any smooth solution. Once the bubble thickness is resolved, the equations of motion do not allow $O(4)$ symmetric solutions joining the two branches. We conclude that the thin wall instanton is unphysical, and that one cannot have processes connecting the two branches, unless negative tension bubble walls are introduced. This also suggests that the self-accelerating branch does not decay into the conventional branch nucleating bubbles. We comment on other kinds of bubbles that could interpolate between the two branches.
\end{abstract}

\section{INTRODUCTION}

The acceleration of the universe is one of the most important problems in modern cosmology. An interesting approach to explain it is to modify gravity at very large distances. One of the most studied examples of a modified gravity model is the Dvali-Gabadadze-Porrati (DGP) brane-world model [1]. In this model, there exits a self-accelerating (SA) solution in which the universe undergoes an exponential expansion without introducing a cosmological constant 2]. However, this branch contains a ghost in the spectrum at the quadratic order [3, 4, 5, 6, 6, 8, 9]. It has been argued [10, 11] that a perturbative argument is not conclusive because the brane is in a strongly coupled regime in this branch, and it is not clear that this implies an instability (see also the recent analysis of nonlinearities in a cosmological background given in Ref. [12]).

There is some indication that the SA branch is pathological at the nonperturbative level [13], coming from the known exact solutions for domain walls [14] and pointlike sources [15] localized on the brane (although the bulk solution is not known in the latter case). In both cases, the effective gravitational mass (or tension) of the source is negative from the five dimensional point of view, which might signal an instability in the full nonlinear theory. This raises a number of interesting questions. If the SA branch contains a ghost instability at nonperturbative level, then we are naturally lead to ask what does this solution decay to. Is it possible to have transitions from one branch to the other? This is what we shall address in

\footnotetext{
*e-mail: ksuke@tap.scphys.kyoto-u.ac.jp

${ }_{\dagger}^{\dagger}$ e-mail: kazuya.koyama@port.ac.uk

‡e-mail: pujolas@ccpp.nyu.edu

$\S$ e-mail: tama@tap.scphys.kyoto-u.ac.jp
}

this paper, with the hope that it can shed some light on the presence of ghosts in the SA solution and the question to what extent they are harmful.

The $5 \mathrm{D}$ action describing the DGP model is given by

$$
S=\int d^{5} x \sqrt{-g} \frac{{ }^{(5)} R}{2 \kappa^{2}}+\int d^{4} x \sqrt{-\gamma}\left(\frac{R}{2 \kappa_{4}^{2}}+\frac{K_{\mu}^{\mu}}{\kappa^{2}}-\sigma\right),
$$

where $\sigma$ is the brane tension and $K_{\mu}^{\mu}$ is the trace of the extrinsic curvature. We assume $Z_{2}$ symmetry across the brane, and above we omitted the matter Lagrangian. This model has two very interesting features. The first is that gravity becomes five dimensional at distances larger than the crossover scale

$$
r_{c}=\frac{\kappa^{2}}{2 \kappa_{4}^{2}}
$$

The other is that for generic sources, it allows two branches. For example, considering only the brane tension $\sigma$, the flat FRW solutions can take two different values of Hubble parameter on the brane given by [2]

$$
H_{ \pm}=\left|K_{ \pm}\right|
$$

with

$$
K_{ \pm}=\frac{1 \pm \sqrt{1+4 r_{c}^{2} \kappa_{4}^{2} \sigma / 3}}{2 r_{c}}
$$

where $K_{ \pm}$is quarter the trace of the extrinsic curvature of the brane in the respective branches. The difference between the two branches can be visualized (for $\sigma>0$ ) as the different embeddings of the brane. In both solutions, the brane is a $4 \mathrm{D}$ de Sitter hyperboloid in the 5D Minkowski spacetime. For the conventional (selfaccelerating) branch, the bulk is identified as the interior (exterior) of the hyperboloid. The SA solution is the one with the larger Hubble rate, $H_{+}$. It is phenomenologically interesting that the Hubble parameter at late times 
in the SA branch approaches a constant $H=1 / r_{c}$ even for vanishing $\sigma$, mimicking a cosmological constant [2].

As has been pointed out in Refs. 3, 4, 5, 6, 7, 8], the SA universe contains a ghost. For a positive tension $\left(H r_{c}>1\right)$, one has a spin-2 graviton having mass in the range $0<m^{2}<2 H^{2}$ and its helicity- 0 excitation is a ghost [5]. On the other hand, for a negative tension brane $\left(H r_{c}<1\right)$, the spin-0 mode becomes a ghost. In the SA universe $\left(H r_{c}=1\right)$, the spin-0 mode and a helicity0 excitation of the spin- 2 mode mix and a ghost arises from the mixing [6]. It was shown that the ghost cannot be removed even in the presence of the second brane in the bulk due to the correlation between spin- 0 and spin- 2 ghosts [9]. On the other hand, the conventional branch of solutions does not contain ghosts, though it does not self-inflate.

Having a ghost in the spectrum for the SA universe, one expects that it is unstable to spontaneous particle creation out of the vacuum or to some even more dramatic process. In this article, we try to go one step further in the analysis of the stability of the SA branch by considering nonperturbative decay channels. Since the conventional branch solution is stable, it is tempting to think that the SA solution decays into the conventional branch solution. Here we shall consider the nucleation of bubbles of the conventional branch in the environment of the SA branch. This would resemble a kind of false vacuum decay in de Sitter space. False vacuum decay is described by an instanton which is a classical solution in an Euclidean time connecting initial and final configurations. In our case, we are interested in a solution that interpolates between the SA and the conventional branches [16].

We shall see that in the thin wall approximation, there exists a solution similar to the Coleman de Luccia instanton [17 describing this process. Unlike the bubble of nothing [18], mediating the decay of the Kaluza-Klein vacuum, the bubbles do not occur spontaneously in the gravitational sector in the DGP model. Rather, a material wall separating the two phases is required for the instanton to exist.

The second part of this paper is dedicated to show that this solution cannot be recovered as a limit of any microscopic model of the bubble based on a scalar field. We interpret this as an indication that the thin wall instanton is unphysical. Hence, the SA branch does not decay nucleating bubbles of the conventional branch. In Section IV. we comment on other possible decay channels.

\section{INSTANTON IN THE THIN WALL APPROXIMATION}

Let us construct the instanton representing a bubble of the conventional branch in the background of the SA branch. We follow the method of Refs. [19], which was recently applied to the analysis of domain wall in the DGP model [14]. However, our result is different from
Refs. [19] in the calculation of the matching condition on the bubble wall, as was also pointed out by Ref. [14].

The bulk spacetime is the 5 D Minkowski spacetime. In Euclidean time, the bulk metric is

$$
d s^{2}=d z^{2}+d r^{2}+r^{2} d \Omega^{2},
$$

where $d \Omega^{2}$ denotes the line element on a unit 3-sphere. The trajectory of the brane in this $5 \mathrm{D}$ Euclidean space is specified by two functions $(z(\tau), r(\tau))$, and we can always choose the gauge condition

$$
\dot{z}^{2}+\dot{r}^{2}=1,
$$

where a dot " " represents differentiation with respect to $\tau$. Then, the induced metric on the brane is given by

$$
d s_{4}^{2}=d \tau^{2}+r^{2}(\tau) d \Omega^{2} .
$$

The equations that determine the embedding are Eq. (2.2) and the Israel junction conditions

$$
K_{\nu}^{\mu}-\delta_{\nu}^{\mu} K=-\frac{\kappa^{2}}{2}\left(T_{\nu}^{\mu}-\frac{1}{\kappa_{4}^{2}} G_{\nu}^{\mu}\right) .
$$

The $(\tau, \tau)$-component of this equation becomes

$$
6 \frac{\dot{z}}{r}=\kappa^{2}\left(\sigma-\frac{3}{\kappa_{4}^{2}} \frac{\dot{z}^{2}}{r^{2}}\right) .
$$

The solution to Eq. (2.2) and Eq. (2.5) is

$$
\begin{aligned}
& r_{ \pm}(\tau)=K_{ \pm}^{-1} \sin \left[K_{ \pm}\left(\tau_{ \pm}^{0}+\tau\right)\right], \\
& z_{ \pm}(\tau)=K_{ \pm}^{-1} \cos \left[K_{ \pm}\left(\tau_{ \pm}^{0}+\tau\right)\right]+z_{ \pm}^{0},
\end{aligned}
$$

where the $+(-)$ subscript refers to the form of the solution in the self-accelerating (conventional) branch segment, and holds for $\tau>0(\tau<0)$. When the extrinsic curvature is positive (negative), the exterior (interior) of the sphere is the bulk. Therefore, when $\dot{z}>0(\dot{z}<0)$, the bulk is the region where the value of $r$ is smaller (larger) than the one on the brane. With this choice, the bulk is guaranteed to be consistently connected on the same side of the brane at the interface of the different segments. One of the constants $\tau_{ \pm}^{0}$ is fixed by the junction condition on the bubble wall (see below) and the other by continuity. Similarly, one of the $z_{ \pm}^{0}$ is fixed by continuity and the other can be set to zero with no loss of generality. Fig. 1 shows the behaviour of these solutions in $(z, r)$ plane.

The continuity of $r$ at $\tau=0$ requires

$$
\frac{\sin \theta_{+}}{K_{+}}=\frac{\sin \theta_{-}}{K_{-}}
$$

where $\theta_{ \pm}=K_{ \pm} \tau_{ \pm}^{0}$. The angular components of the junction condition (2.4) have a delta function singularity at $\tau=0$ as $\dot{r}$ is discontinuous. The singular part of the junction condition close to $\tau=0$ is given by

$$
2 \frac{\ddot{r}}{\dot{z}}=-\kappa^{2}\left(\mu \delta(\tau)+\frac{2}{\kappa_{4}^{2}} \frac{\ddot{r}}{r}\right) .
$$




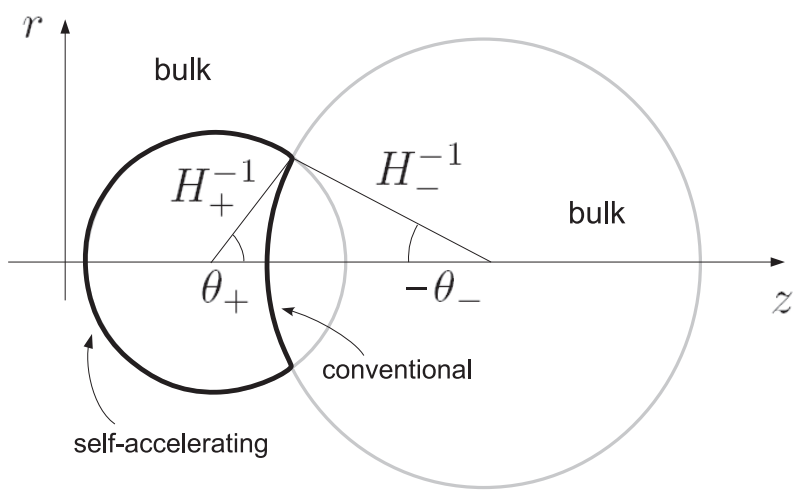

FIG. 1: Trajectory of the brane in $(z, r)$ plane (for $\sigma>0$ ).

The term on the left-hand side of the above equation is simply written as $2 \dot{\theta}$ by introducing the parametrization $\dot{r}=\cos \theta$ and $\dot{z}=-\sin \theta$. Integrating over the infinitesimal interval around $\tau=0$, and using Eq. (2.6), we obtain the following jump condition on the bubble

$$
2\left(\theta_{+}-\theta_{-}\right)+4 r_{c}\left(K_{+} \cot \theta_{+}-K_{-} \cot \theta_{-}\right)=-\kappa^{2} \mu,
$$

where $\mu$ is a tension of the bubble wall. The first term is the deficit angle arising from the 5D Einstein tensor and the second term is the jump of the $3 \mathrm{D}$ extrinsic curvature across the bubble wall coming from the 4D Einstein tensor. $^{1}$

The second term on the left-hand side can be rewritten as

$$
\begin{aligned}
r_{c}\left(K_{+} \cot \theta_{+}-K_{-} \cot \theta_{-}\right) \\
\quad=-r_{c} \frac{K_{i}}{\sin \theta_{i}}\left(\sin \theta_{+}+\sin \theta_{-}\right) \tan \frac{\theta_{+}-\theta_{-}}{2} \\
\quad=-\tan \frac{\theta_{+}-\theta_{-}}{2},
\end{aligned}
$$

where we used the identity

$$
\frac{\cos \theta_{+}-\cos \theta_{-}}{\sin \theta_{+}+\sin \theta_{-}}=-\tan \frac{\theta_{+}-\theta_{-}}{2},
$$

and $r_{c}\left(K_{+}+K_{-}\right)=1$, which immediately follows from Eq. (1.3). Thus we obtain an equation for $\bar{\theta} \equiv\left(\theta_{+}-\right.$ $\left.\theta_{-}\right) / 2$,

$$
(\tan \bar{\theta})-\bar{\theta}=\frac{\kappa^{2} \mu}{4},
$$

which directly determines the deficit angle, $-4 \bar{\theta}$. If there is no domain wall tension, Eq. (2.11) immediately implies that there is no instanton that describes the transition from the SA universe to the conventional branch

\footnotetext{
${ }^{1}$ Note that in Refs. [19], the integration of the left-hand side of Eq. 2.8 was done with $\dot{z}$ fixed. This is not true and thus our result for the 5D singular part is different from Refs. [19] (see also Ref. 14]).
}

solutions. Thus the SA universe cannot decay into the conventional branch solution without introducing a domain wall tension. On the other hand, Eq. (2.11) admits a solution if we have the positive tension wall $\mu>0$. This would indicate that a 2-brane can induce the SA universe to decay into the conventional branch.

When both $\kappa^{2} \mu$ and $\kappa_{4}^{2} r_{c}^{2} \sigma$ are small, $\left|\theta_{-}\right| \ll \theta_{+} \ll 1$ holds. In this case, we have

$$
\theta_{+} \simeq\left(6 \kappa^{2} \mu\right)^{1 / 3}
$$

The difference between the instanton and the background action is

$$
\Delta I \approx 12 \pi^{2} \frac{\theta_{+}^{4} r_{c}^{3}}{\kappa^{2}} .
$$

The suppression of the rate of this tunnelling process is of order $\sim e^{-\Delta I}$. Thus, bubbles with a low enough tension, as given by

$$
\kappa^{2} \mu \ll\left(r_{c} / \kappa_{4}\right)^{-3 / 2},
$$

have an unsuppressed tunnelling rate. For $r_{c}$ of order of the present Hubble radius we have $r_{c} / \kappa_{4} \approx 10^{60}$, which leads to a tension of order $10^{-90} \kappa^{-2}$ or less.

\section{NON-EXISTENCE OF SMOOTH BRANCH-CHANGING SOLUTIONS}

Let us now show that any attempt to find a smooth version of the instanton of the previous section which interpolates between the two branches is doomed to fail. We shall assume that the bubble is supported by a scalar field $\phi$ localized on the brane, with some potential $V(\phi)$ which we do not need to specify. Generalizing a constant $\sigma$ to a function $\rho_{E}$, Eqs. (2.2) and (2.5) are more conveniently written in this case as

$$
-\frac{\dot{z}}{r}=\hat{K}_{ \pm}\left(\rho_{E}\right)
$$

and

$$
\frac{1-\dot{r}^{2}}{r^{2}}=\hat{K}_{ \pm}^{2}\left(\rho_{E}\right)
$$

where

$$
\hat{K}_{ \pm}\left(\rho_{E}\right) \equiv \frac{1 \pm \sqrt{1+4 r_{c}^{2} \kappa_{4}^{2} \rho_{E} / 3}}{2 r_{c}}
$$

and the "Euclidean energy density" is given by ${ }^{2}$

$$
\rho_{E}=-\frac{1}{2} \dot{\phi}^{2}+V(\phi) \text {. }
$$

2 It is manifest that the positivity of $\rho_{E}$ is not guaranteed in general even for a normal field as is clear from Eq. 3.4. Here we used the notation $\rho_{E}$ from the analogy to the closed FRW model, but its analytic continuation to the Lorentzian region 


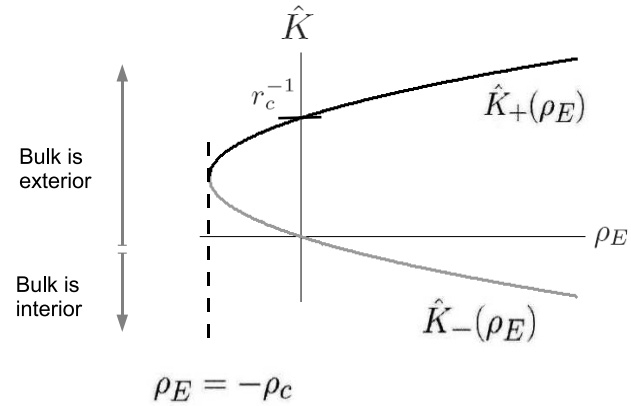

FIG. 2: Relation between $\rho_{E}$ and $\hat{K}$. we do not have to consider dashed line. Any solution that smoothly joins the two branches must have an energy density $\rho_{E}=-\rho_{c}$ at some point.

We have chosen "-" sign on the left-hand side in Eq. (3.1). This sign is conventional, but once we choose it we have to keep it fixed during the calculation. It is clear from (3.3) that a solution that smoothly connects the two branches at some point must have $\rho=-\rho_{c}$, where

$$
\rho_{c} \equiv \frac{3}{4 \kappa_{4}^{2} r_{c}^{2}} .
$$

Even if we allow for negative value of $\rho_{E}$, we can easily show that there are no branch-changing solutions. In order to interpolate between the two branches, $\rho_{E}$ has to reach the value $-\rho_{c}$, as shown in Fig. 2 . Hence, $\dot{\rho}_{E}$ must change sign at the point where $\rho=-\rho_{c}$. On the other hand, the equation of motion for $\phi$ is equivalent (as long as $\dot{\phi} \neq 0)$ to

$$
\dot{\rho}_{E}=3 \frac{\dot{r}}{r} \dot{\phi}^{2}
$$

This means that $\dot{\rho}_{E}$ can only change sign if $\dot{r}$ also does, and consequently that if $\rho_{E}$ develops a minimum, then $r(\tau)$ also does. Given that the minimum of $\rho_{E}$ must be at $-\rho_{c}$, Eqs. (3.2) and (3.3) imply that the minimum in $r(\tau)$ occurs with $r=2 r_{c}$, as illustrated in Fig. 3. The key point is that having a minimum at this value is incompatible with Eq. (3.2), when we try to integrate it with the choice of sign corresponding to the SA branch. Indeed, if $r(\tau)$ has a minimum at $2 r_{c}$, then the left-hand side of Eq. (3.2) is bounded above by $1 /\left(2 r_{c}\right)^{2}$. But in

does not mean the energy density in general. When the expression is analytically continued through the $\tau=$ constant maximal hypersurface given by $\dot{r}(\tau)=0$, the analytic continuation of $\rho_{E}$ becomes the energy density. In the context of the bubble nucleation, however, the hypersurface corresponding to the junction between Euclidean and Lorentzian regions is not given by a $\tau=$ constant surface. In this case, the analytic continuation of $\rho_{E}$ becomes minus the pressure along the direction orthogonal to the bubble wall, which is not directly related to the wall tension.
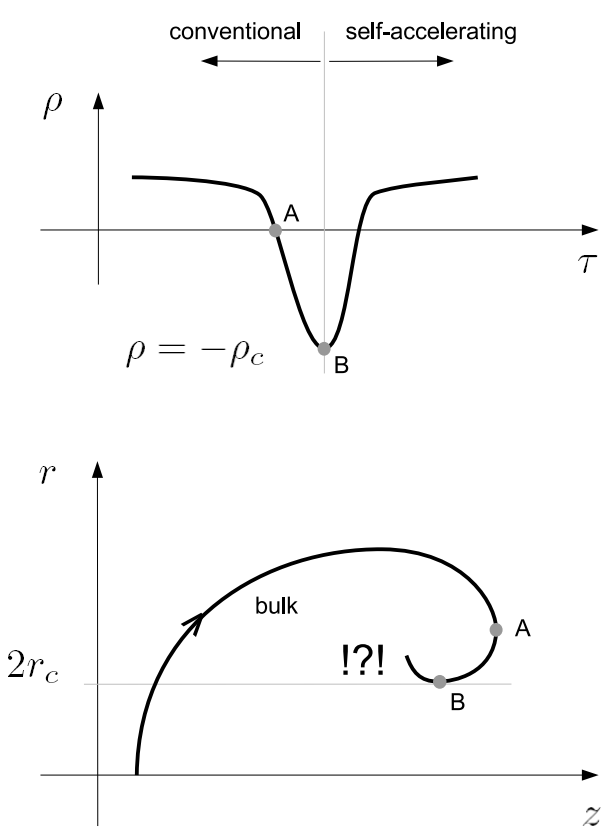

FIG. 3: In the upper panel we sketch the form of $\rho_{E}(\tau)$ for a solution that smoothly joins the two branches. At the point $B$, we have $\rho=-\rho_{c}$. In the lower panel, we show schematically the trajectory starting from the conventional branch. At point $A, \rho_{E}$ vanishes, and so does the extrinsic curvature. The bulk is the interior $(r<r(\tau))$ before that point and the exterior $(r>r(\tau))$ after it. At point $B$, by the conservation equation, $r(\tau)$ develops a minimum at $2 r_{c}$. However, matching to the $\mathrm{SA}$ branch at point $B$ is incompatible with Eq. (3.2).

the SA branch we have $\hat{K}_{+}^{2}>1 /\left(2 r_{c}\right)^{2}$. This is a contradiction. Note that this proof does not rely on any assumptions of the regularity of the instanton (e.g., at the poles, $r=0$ ), so even if singular solutions are allowed in the path integral, they would not include an $O(4)$ symmetric branch-changing configuration. Hence, we conclude that there is no smooth solution connecting two different branches.

By contrast, if and only if we stay on the conventional branch on both sides of the point at which $\rho=-\rho_{c}$, the equations for the brane trajectory can be integrated around the minimum. This is the only possible configuration compatible with the profile of $\rho(\tau)$ shown in upper panel of Fig. 3 .

In summary, the argument for the absence of branchchanging solutions consists of two observations. The first is that, in order to join the two branches, $\rho_{E}(\tau)$ must have a minimum at $-\rho_{c}$. The second is that a minimum in $\rho_{E}(\tau)$ is only compatible with the equations of motion if we stay on the conventional branch around the minimum.

It is instructive to compare this situation to what we would find in GR under the assumption that $\rho_{E}(\tau)$ has a 
minimum. By the conservation equation (3.6), one also concludes that $r(\tau)$ has a minimum. In GR, we would not have Eq (3.1), and $\hat{K}_{ \pm}^{2}$ in Eq. (3.2) would be replaced by $\kappa_{4}^{2} \rho_{E} / 3$. Since this is always increasing in $\rho_{E}$, it follows by the same argument that a minimum in $r(\tau)$ would be incompatible with Eq. (3.2) (irrespective of the value of $\rho_{E}$ at the minimum). Hence, the reason why one can obtain a minimum in DGP for the conventional branch relies on the very peculiar property that

$$
\frac{\partial \hat{K}_{-}^{2}\left(\rho_{E}\right)}{\partial \rho_{E}}<0
$$

in the range $-\rho_{c}<\rho_{E}<0$. The Hubble rate in the closed FRW universe is given by $H^{2}=\hat{K}_{-}^{2}(\rho)$ where $\rho$ is the energy density. Hence, in this range $H^{2}$ increases with decreasing the energy density $\rho$, which is a manifestation of anti-gravity. This is expected since for very low densities gravity in DGP behaves as in $5 \mathrm{D}$, where the sign of the effective Newton constant is reversed for $\rho_{E}<0$.

The obstruction to find a minimum arises because Eq. (3.4) enforces that the signs of $\dot{r}$ and $\dot{\rho}_{E}$ are identical. Generally, this relation holds if $\rho_{E}+P_{E}<0$, where $P_{E}$ is the analytic continuation of the pressure to Euclidean. The energy momentum tensor on the four dimensional brane takes the form, $T_{\nu}^{\mu}=\operatorname{diag}\left(-\rho_{E}, P_{E}, P_{E}, P_{E}\right)$. In the case of a scalar field, we have $P_{E}=-\dot{\phi}^{2} / 2-V(\phi)$. As $P_{E}$ dominates in the thin wall case, the above condition $\rho_{E}+P_{E}<0$ is approximately interpreted as the negative pressure of the bubble wall, which means that the tension of the bubble wall is positive. Then, permitting the presence of a negative tension bubble drastically alters our discussion. In particular, it is not difficult to construct branch-changing instantons with a pair of positive and negative tension bubbles. However, a normal scalar field cannot support a negative tension bubble. The only way to incorporate a negative tension bubble using a scalar field model is to flip the overall sign of its action, i.e. to introduce a ghost. In general, it seems that the negative tension bubble requires the presence of an additional ghost matter sector ${ }^{3}$. Therefore we shall not pursue this direction here. (See Ref. [20] for a recent discussion on the nucleation of pairs of ghost and normal domain walls.)

\section{DISCUSSION}

In summary, we have studied the possibility to construct an $O(4)$ invariant instanton that describes the nucleation of bubbles of the conventional branch in the self-

\footnotetext{
3 This ghost should be distinguished from the familiar ghost which resides in the gravitational sector in the SA branch. The gravitational ghost is built into the DGP model from the beginning, and it is already taken into account in our present analysis.
}

accelerating (SA) universe. We found that this is impossible unless there is a material bubble wall which induces the transition. Introducing the bubble wall tension, one can find an instanton in the thin wall limit. However, we showed that it is impossible to construct this instanton in a scalar field model of the wall. The reason is that at the point where the two branches are connected, the equations of motion cannot be integrated in the SA branch. It immediately follows that the thin wall instanton is unphysical and that transitions between the two branches cannot happen (at least with $O(4)$ symmetry).

We should emphasize that the obstruction to have the solution is not due to the impossibility to embed the brane in such a way that the bulk is the exterior of the brane on one side of the wall and the interior on the other ${ }^{4}$. Instead, it is a direct consequence of the equivalent of the null energy condition $\left(\rho_{E}+P_{E}<0\right)$, which is satisfied in a scalar model of the bubble. Let us now give a few comments on the interpretation of our results.

\section{Bubbles in the ' $\pi$-Lagrangian'}

It is also useful to analyze the bubble configurations in terms of the effective scalar field Lagrangian introduced in Ref. 21] (see also Ref. 22] and 23]) to describe the decoupling limit. In this limit, the most relevant nonlinearities are encoded in the brane bending mode $\pi$, and are described by the equation of motion

$$
3 \square \pi+\frac{(\square \pi)^{2}-\left(\nabla_{\mu} \nabla_{\nu} \pi\right)^{2}}{\Lambda^{3}}=-\kappa_{4} \frac{T}{2},
$$

where $\Lambda^{3}=\left(2 \kappa_{4} r_{c}^{2}\right)^{-1}$ and $T$ is the trace of the stress tensor of $\phi$. In this treatment, the equation of motion for $\phi$ is the same as in flat space. In the absence of the brane tension, the conventional branch is $\pi_{0}=$ const and the SA branch is $\pi_{0}=-\left(\Lambda^{3} / 2\right) x^{\mu} x_{\mu}$. One expects that analog of the thin wall instanton of Sec. II is

$$
\pi=-\left(\Lambda^{3} / 2\right)\left(\xi^{2}-\xi_{0}^{2}\right) \Theta\left(\xi^{2}-\xi_{0}^{2}\right),
$$

where we introduced the 'Rindler' coordinate $\xi=$ $\left(x^{\mu} x_{\mu}\right)^{1 / 2}$. Inside the hyperboloid at $\xi=\xi_{0}$ there is the conventional branch and outside the SA branch. Clearly, the only nontrivial step to check whether this is a solution of Eq. (4.1) is to see what happens on the hyperboloid. Assuming that $\pi$ depends only on $\xi$, the left-hand side of Eq. (4.1) is rewritten as

$$
\left(1+\frac{2}{\Lambda^{3}} \frac{\pi^{\prime}}{\xi}\right) \pi^{\prime \prime}+\left(3+\frac{2}{\Lambda^{3}} \frac{\pi^{\prime}}{\xi}\right) \frac{\pi^{\prime}}{\xi}
$$

\footnotetext{
4 To illustrate this point, consider a potential $V(\phi)$ with a 'false' vacuum with positive cosmological constant $(\mathrm{CC})$ and a 'true' vacuum with negative $C C$ (but larger than $-\rho_{c}$ ). Then, true vacuum bubbles solutions within the same branch can form. For the conventional branch, the instanton looks like Fig. 1 with the bulk being the complement of what is shown there, so that the bulk corresponds the interior or the exterior of the brane in either side of the bubble wall.
} 
where $\pi^{\prime}=\partial_{\xi} \pi$. Hence, the singular term at $\xi=$ $\xi_{0}$ on the left-hand side has a distributional form like $\operatorname{sign}\left(\xi-\xi_{0}\right) \delta\left(\xi-\xi_{0}\right)$ (and it is proportional to $\xi_{0}$ ). This means that the configuration (4.2) cannot be sourced by a simple $\delta$-function. In fact, integrating around the bubble wall, one obtains

$$
\Delta \pi^{\prime}+\frac{1}{\Lambda^{3} \xi_{0}} \Delta\left(\pi^{\prime}\right)^{2},
$$

where $\Delta \pi^{\prime}=\left.\pi^{\prime}\right|_{\mathrm{SA}}-\left.\pi^{\prime}\right|_{\text {conventional. }}$ Given that $\pi^{\prime}=-\Lambda^{3} \xi$ in the SA branch, this is identically zero! This does not mean that this is a 'vacuum' (no source) solution, but rather that the total tension of the source vanishes, as would happen for example in a system of two walls with opposite tensions. The fact that the two terms in the previous equation cancel out is the remnant of the cancellation between the terms linear in $\bar{\theta}$ on the left-hand side of Eq. (2.11). Recall that these terms originate from the intrinsic curvature and the extrinsic curvature, respectively. In the exact solution (2.11), these terms do not cancel out at higher order in $\bar{\theta}$, and this is what allows to have solutions with a net bubble wall tension. Hence, the $\pi$ Lagrangian does not reproduce the relation between the deficit angle and the tension (2.12) for the thin wall approximation. Interestingly enough, it does reproduce the fact that, in order to have a branch-changing solution, one has to provide a negative energy density at some point.

\section{Strong coupling}

If the branch-changing instanton existed, in principle there would be the concern of how good an approximation to the nucleation process it is, given that the brane would be at an infinitely strongly coupled regime at the branch-changing point ${ }^{5}$. One could expect that any perturbation would produce a large backreaction, so that the actual process significantly differs from the solution.

However, even if the infinitely strong coupling is achieved at the branch-changing point, it may not prevent the solution from having a physical meaning. The present situation is quite similar to the violation of the WKB approximation in false vacuum decay. At the turning points, the WKB approximation is broken maximally. However, in the description of the bubble nucleation, the time coordinate is extended from the real (or the imaginary) axis to the full complex plane. Then the WKB approximation is violated only around a point, e.g. $t=0$, in

\footnotetext{
5 The infinite strong coupling can be easily seen in terms of the effective field theory 4.1. Consider for simplicity that the only source is the brane tension $\sigma$. The de Sitter invariant solutions are $\pi_{0}=(c / 2) x^{\mu} x_{\mu}$, with $c=-K_{ \pm} r_{c} \Lambda^{3}$ and $K_{ \pm}$given by (3.3), and represent the de Sitter branes in the conventional or SA branch. The fluctuations around this background can be studied by plugging $\pi=\pi_{0}+\delta \pi$ in 4.1), and one obtains that the coefficient in front of the kinetic term for $\delta \pi$ is $\left(1-2 K_{ \pm} r_{c}\right)$. Hence, this mode becomes infinitely strongly coupled for $K_{ \pm} r_{c}=$ $1 / 2$, which is precisely the point where the two branches join, $\rho=-\rho_{c}$.
}

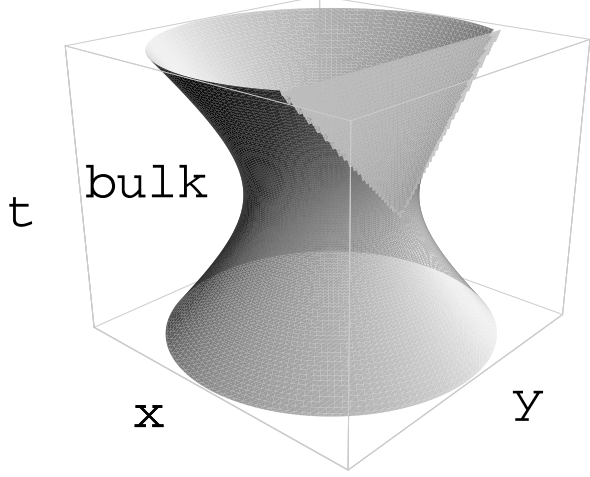

FIG. 4: Null bubble in the full DGP model. The two branches are connected on a light cone. The upper half of this geometry is given by the continuation of the instanton in Fig. 1 in the limit when the bubble wall tension vanishes. The lower half is the 'background' solution, given by the SA branch. In the $\pi$ language, it corresponds to the solution (4.3).

the complex plane. The situation concerning the strong coupling seems to have the same structure in the complex plane. Therefore having a region with strong coupling would not render the analysis of instantons invalid. This issue clearly deserves further investigation, though.

Let us emphasize that our main conclusion that there is no evidence for the instability through the branch-changing bubble nucleation is not affected by these considerations. What we found is that there is no branch-changing configuration even at the level of a classical solution to the Euclidean equations of motion. In contrast, note that the DGP model does allow for solutions that are infinitely strongly coupled on one time slice, although they stay in the conventional branch at all times $^{6}$. Hence, in this sense, there is a clear asymmetry between the two branches. This suggests that there is a deep reason for it, and it might be tempting to associate it with the presence of ghosts in the SA branch.

\section{Zero radius bubble}

Another interesting solution is the "zero-radius" instanton $^{7}$. In Sec. II we obtained an instanton solution with a positive tension thin wall. Although we have shown that this thin wall instanton cannot be understood as a physically meaningful solution in Sec. III, its zero-radius limit requires more careful consideration. As the bubble radius shrinks, the tension of the wall goes to zero. Hence, the zero-radius instanton does not seem to require any matter field. In the Euclidean space, the solution is a trivial sphere except for one point. However, its an-

\footnotetext{
6 The topology of the brane in such a solution is not $S^{4}$ but $S^{1} \times S^{3}$ as long as a bubble wall composed of a scalar field is concerned. 7 We thank Jaume Garriga for bringing our attention to this limit.
} 
alytic continuation to the Lorentzian region describes a formation of a bubble of the conventional branch from a single spacetime point. The junction of the two branches is given by a null hypersurface as shown in Fig. 4. Although we call this solution an instanton, the difference between the instanton action and the background one vanishes. Therefore one can say that there is no barrier for this process. Namely, the process could occur classical mechanically if the solution turned out to be physical.

It is not clear whether this instanton configuration solves the equations of motion in the DGP model or not. To answer this question, we should carefully study the junction at the null hypersurface ${ }^{8}$. However, this is not what we wish to address here.

In fact, the inverse process from the conventional branch to the SA branch as illustrated in Fig. 5 also seems to exist similarly. Hence, if this type of solutions is physical, the consistency of the DGP model in the conventional branch is compromised. The point that we want to stress is that, even if the zero-radius instanton solves the equations of motion, it is far from clear if it has any physical meaning.

In contrast to the case of the strong coupling issue that we discussed above, we would say that the zeroradius instanton is clearly outside the range of validity of the present effective theory at least at the classical level. The DGP model has a strong coupling length scale below which one cannot trust this effective theory. Here, the zero-radius instanton contains a jump in its metric, and we know that this jump cannot be understood as a limit of any smoothed configurations. Hence, the configuration of the zero-radius instanton is clearly outside the range of validity of the effective theory.

Furthermore, if we accept this solution as a physically meaningful one, classical initial-value problems become non-deterministic. Namely, the future evolution of a given initial data has various possibilities ${ }^{9}$. Such a situ-

8 Again, it is possible to understand this solution using the effective theory for the brane bending mode $\pi$. The non-linear equation (4.1) admits a solution of the form

$$
\pi=-\left(\Lambda^{3} / 2\right) x^{\mu} x_{\mu}\left(1-\Theta\left(-x^{\mu} x_{\mu}\right) \Theta\left(x^{0}\right)\right),
$$

where $\Theta$ is a Heaviside step function. In the interior of the future light cone, $x^{\mu} x_{\mu}<0$ and $x^{0}>0$, the solution corresponds to the conventional branch and the remaining part is in the SA branch. (Similar solutions with the bubble restricted to $x^{0}>0$ can also be constructed in the presence of the brane tension.) The only non-trivial part of 4.3 is what happens at the light cone and at the origin. Besides the origin, one can easily see that the localized distributional terms appearing in the equation of motion are of the form $\xi \delta(\xi), \xi^{2} \delta^{\prime}(\xi)$ and $(\xi \delta(\xi))^{2}$ where $\xi=\left(x^{\mu} x_{\mu}\right)^{1 / 2}$. While the first two can be taken to be zero in a distributional sense, it is not completely clear that one can set $(\xi \delta(\xi))^{2}$ to zero. However, if we use a representation of the delta function defined as a limit of a function, e.g. $\delta(\xi) \equiv \lim _{\epsilon \rightarrow 0}(\sqrt{2 \pi} \epsilon) \exp \left(-\xi^{2} / 2 \epsilon^{2}\right)$, this term vanishes. Up to this subtlety, this is a solution of the $\pi$ Lagrangian.

9 A similar situation was found for 'stealth' branes [24].

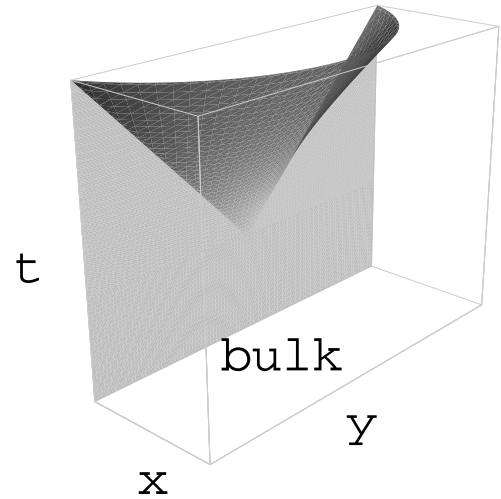

FIG. 5: Inverse null bubble in the DGP model. In this configuration, the brane is in the SA branch in the interior of the future light cone of some event.

ation should not be allowed when we consider not a low energy effective theory but a complete theory. In an appropriate UV completion for the DGP model, the zeroradius instanton must not be actually a solution. For this reason, we conclude that this zero-radius instanton is excluded from the physical solutions at the classical level.

One may still think that this instanton has some meaning as a quantum process. One is then lead to the logic that we used above regarding the issue of the strong coupling for hypothetical smooth instanton solutions. There, we extended the time coordinate to the full complex plane. Once we do so, it may look unclear whether the tunnelling process described by this instanton is really forbidden or not. However, since the zero-radius instanton is not analytic, containing a step function, its analytic continuation to the complex valued time does not make sense. Therefore the regularization method with the aid of the complex valued time does not work for the zeroradius instanton. So, it is not clear if the configurations in Fig. 4 and Fig. 5 have a physical meaning even in this sense.

Bubbles in the bulk

Let us mention that another possible instability is the nucleation of SA branes within the bulk, resembling bubbles of nothing. This process was considered in [25]. The nucleated SA branes would eat up the bulk and eventually collide with the other (our) brane. This can happen irrespective of the branch in which our brane is and for any value of its tension. This suggests that this is not related to the perturbative ghost instability, for the conventional branch does not have any ghost mode. Furthermore, if the brane tension is negative, one can have a similar instability due to the the nucleation of conventional branch bubbles in the bulk.

In Ref. 25], the decay rate was estimated as $\sim \exp \left(-r_{c}^{2} / \kappa_{4}^{2}\right)$ when the brane tension is small (a similar instability was discussed in Ref. 24] when 
the $Z_{2}$ symmetry across the branes is not imposed). Even though this process is suppressed, it represents a catastrophic instability if the light cone extends into the bulk forever in the past. However, once we introduce a cutoff in time, typically of order the age of the Universe $H_{0}^{-1} \sim r_{c}$, the probability that an observer is hit by such a SA brane nucleated in the bulk becomes really suppressed. Let us just add that one can also avoid this instability trivially by restricting to topology preserving configurations.

\section{Taming the ghost?}

It is still unclear what is the consequence of the ghost instability in the SA universe. Usually, the ghost leads to spontaneous pair creation of ghost and normal particles. Once such a channel opens, Lorentz invariance leads to a divergence of the particle creation rates. If we consider the situation in which there is a spin-2 ghost, we need to treat the helicity zero mode in a different way. Otherwise, negative norm states appear. But if we take a different prescription for the quantization for the helicity zero mode, this procedure necessarily breaks de Sitter invariance. When there is a spin-0 ghost, a similar phenomenon happens. In this case, the mass of the ghost is given by $-4 H^{2}$. But we know that there is no de Sitter invariant vacuum state for a scalar field with negative mass squared. Once de Sitter invariance is broken, one may be allowed to consider the possibility that the non-covariant cutoff scale may arise due to the strong coupling effect. Then the particle creation could be milder than the usual ghost in the Minkowski background. Further studies are necessary to verify this, but together with the non-existence of the non-perturbative instability, the ghost in the SA universe could be less harmful than expected.

\section{Acknowledgments}

We would like to thank Gia Dvali, Gregory Gabadadze, Jaume Garriga, Alan Guth, Nemanja Kaloper, Matt Kleban, Michele Redi and Alex Vilenkin for useful discussions. KK is supported by PPARC/STFC. OP acknowledges support from DURSI (Generalitat de Catalunya), under contract 2005 BP-A 10131. TT is partially supported by Monbukagakusho Grant-in-Aid for Scientific Research Nos. 17340075 and 19540285 . This work is also supported in part by the 21st Century COE "Center for Diversity and Universality in Physics" at Kyoto university, from the Ministry of Education, Culture, Sports, Science and Technology of Japan, and by the Japan-U.K. Research Cooperative Program both from Japan Society for Promotion of Science.
[1] G. R. Dvali, G. Gabadadze and M. Porrati, Phys. Lett. B 484, 112 (2000) arXiv:hep-th/0002190.

[2] C. Deffayet, Phys. Lett. B 502, 199 (2001) arXiv:hep-th/0010186.

[3] M. A. Luty, M. Porrati and R. Rattazzi, JHEP 0309 (2003) 029 arXiv:hep-th/0303116;

[4] A. Nicolis and R. Rattazzi, JHEP 0406 (2004) 059 arXiv:hep-th/0404159;

[5] K. Koyama and K. Koyama, Phys. Rev. D 72 (2005) 043511 arXiv:hep-th/0501232; K. Koyama, Phys. Rev. D 72 (2005) 123511 arXiv:hep-th/0503191.

[6] D. Gorbunov, K. Koyama and S. Sibiryakov, Phys. Rev. D 73 (2006) 044016 arXiv:hep-th/0512097.

[7] C. Charmousis, R. Gregory, N. Kaloper and A. Padilla, JHEP 0610 (2006) 066 arXiv:hep-th/0604086.

[8] M. Carena, J. Lykken, M. Park and J. Santiago, Phys. Rev. D 75, 026009 (2007) arXiv:hep-th/0611157.

[9] K. Izumi, K. Koyama and T. Tanaka, arXiv:hep-th/0610282,

[10] C. Deffayet, G. Gabadadze and A. Iglesias, JCAP 0608, 012 (2006) arXiv:hep-th/0607099.

[11] G. Dvali, arXiv:hep-th/0610013

[12] K. Koyama and F. P. Silva, arXiv:hep-th/0702169.

[13] G. Gabadadze, arXiv:hep-th/0612213 arXiv:0705.1929 [hep-th].

[14] G. Dvali, G. Gabadadze, O. Pujolas and R. Rahman, arXiv:hep-th/0612016
[15] G. Gabadadze and A. Iglesias, Phys. Rev. D 72, 084024 (2005) arXiv:hep-th/0407049]; Phys. Lett. B 632, 617 (2006) arXiv:hep-th/0508201.

[16] The way how we interpret a gravitational instanton here is explained in e.g. T. Tanaka and M. Sasaki Prog. Theor. Phys. 88, 503 (1992)

[17] S. R. Coleman and F. De Luccia, Phys. Rev. D 21, 3305 (1980).

[18] E. Witten, Nucl. Phys. B 195, 481 (1982).

[19] R. Gregory and A. Padilla, Phys. Rev. D 65 (2002) 084013 arXiv:hep-th/0104262; R. Gregory and A. Padilla, Class. Quant. Grav. 19 (2002) 279 arXiv:hep-th/0107108.

[20] R. Emparan and J. Garriga, JHEP 0603, 028 (2006) arXiv:hep-th/0512274.

[21] M. A. Luty, M. Porrati and R. Rattazzi, JHEP 0309, 029 (2003) arXiv:hep-th/0303116.

[22] A. Nicolis and R. Rattazzi, JHEP 0406, 059 (2004) arXiv:hep-th/0404159.

[23] G. Gabadadze and A. Iglesias, Phys. Lett. B 639, 88 (2006) arXiv:hep-th/0603199.

[24] R. Cordero and A. Vilenkin, Phys. Rev. D 65, 083519 (2002) arXiv:hep-th/0107175.

[25] N. Kaloper, Phys. Rev. D 71, 086003 (2005) [Erratumibid. D 71, 129905 (2005)] arXiv:hep-th/0502035. 